Mathematical Medicine and Biology (2004) 0, 1-26

doi: 10.1093/imammb/drixxx

\title{
Biphasic behaviour in malignant invasion
}

\author{
BEN P. MARCHANT $* \dagger$ \\ Rothamsted Research, Harpenden, \\ Hertfordshire, AL5 2JQ, UK. \\ JOHN NORBURY $\ddagger$ \\ Mathematical Institute, \\ 24-29 St. Giles', Oxford, OX1 3LB, UK.
}

AND

HELEN M. BYRNE§

Centre for Mathematical Medicine, School of Mathematical Sciences, University of Nottingham, Nottingham, NG7 2RD, UK.

[Received on $\mathrm{xx}$; revised on yy]

Invasion is an important facet of malignant growth that enables tumour cells to colonise adjacent regions of normal tissue. Factors known to influence such invasion include the rate at which the tumour cells produce tissue-degrading molecules, or proteases, and the composition of the surrounding tissue matrix. A common feature of experimental studies is the biphasic dependence of the speed at which the tumour cells invade on properties such as protease production rates and the density of the normal tissue. For example, tumour cells may invade dense tissues at the same speed as they invade less dense tissue, with maximal invasion seen for intermediate tissue densities. In this paper a theoretical model of malignant invasion is developed. The model consists of two coupled partial differential equations describing the behaviour of the tumour cells and the surrounding normal tissue. Numerical methods show that the model exhibits steady travelling wave solutions that are stable and may be smooth or discontinuous. Attention focuses on the more biologically relevant, discontinuous solutions which are characterised by a jump in the tumour cell concentration. The model also reproduces the biphasic dependence of the tumour cell invasion speed on the density of the surrounding normal tissue. We explain how this arises by seeking constant-form travelling wave solutions and applying non-standard phase plane methods to the resulting system of ordinary differential equations. In the phase plane the system possesses a singular curve. Discontinuous solutions may be constructed by connecting trajectories that pass through particular points on the singular curve and recross it via a shock. For certain parameter values there are two points at which trajectories may cross the singular curve and, as a result, two distinct discontinuous solutions may arise.

\section{Introduction}

One of the most insidious features of malignant solid tumours is their ability to invade and colonise regions occupied by healthy tissues and extracellular matrix (ECM). This requires the coordinated making

\footnotetext{
*email: ben.marchant@bbsrc.ac.uk

$\dagger$ author for correspondence

‡email: john.norbury@lincoln.ox.ac.uk

§email: helen.byrne@nottingham.ac.uk
} 
and breaking of cell-cell and cell-ECM contacts and the degradation of ECM components which otherwise obstruct tumour cell migration and invasion. The large body of experimental work devoted to elucidating the mechanisms by which tumours invade underlines its importance in cancer biology. Much of this work is embodied in the three step hypothesis (Stetler-Stevenson et al. (1993)) which characterises a cell's invasive potential on the basis of three inter-related phenomena: adhesion, local proteolysis and migration. A cell's adhesive properties are controlled by the number and type of adhesion molecules on its outer membrane. Normal cells have large numbers of homotypic adhesion molecules which cause the cells to stick to each other. These homotypic bonds are weaker for malignant cells and instead there are stronger heterotypic bonds between the cells and the ECM. Malignant cells that are attached to the ECM can produce enzymes called proteases that degrade components of the ECM in a process known as proteolysis. Having degraded the local ECM the cell may then migrate into the vacant space. The mechanisms by which tumour cells migrate may be divided into three main types: chemokinesis describes random motion in response to a chemical stimulant; chemotaxis describes directed motion in response to concentration gradients of soluble factors; and haptotaxis describes directed motion in response to concentration gradients of bound, insoluble and non-diffusible chemicals present within the ECM. Haptotaxis is assumed to be the dominant migration mechanism within the model described in this paper. We note that chemotaxis has a similar mathematical representation and therefore our results should extend easily to models of chemotactic motion.

While the molecular mechanisms that underpin tumour invasion remain to be characterised fully, it is generally accepted that those associated with cell-cell and cell-matrix adhesion are crucial (Cairns et al. (2003)). For example, adhesion molecules such as integrins and cadherins enable cells to sense and interact with their environment. The expression of these molecules and, hence, tumour cell adhesion and motility are known to be influenced by a variety of factors that are overexpressed in tumours such as liver, breast and colon. These include the plasminogen activation system which, through the urokinase-type plasminogen activator (UPA) and its receptor (UPAR), not only promotes ECM proteolysis by regulating plasminogen activation but also regulates cell-ECM interactions by acting as an adhesion receptor for vitronectin, an ECM component, and by modulating integrin function. In addition, uPA/uPAR regulates cell migration by acting as a signal transduction molecule and through its intrinsic chemotactic activity (Sidenius \& Blasi (2003)).

Like the plasminogen system, the metalloproteases and their inhibitors perform a variety of roles in tumour invasion: through ECM degradation they enable cells to move through tissues; through proteolytic cleavage they release growth factors, making them available to cells at a distance; and, by regulating receptor cleavage, they help to terminate migratory signalling (Chang \& Werb (2001)). There are two closely related metalloprotease families: matrix metalloproteases (MMPs) and metalloproteasedisintegrins (ADAMs). MMPs are characterised by their ability to degrade the ECM and their dependence on zin binding for proteolytic activity. ADAMs are transmembrane proteins that contain disintegrin and metalloprotease domains. Their activity tends to focus on tumour cell adhesion and regulating growth signalling. Tissue inhibitors of metalloproteases (TIMPs) are a family of secreted proteins that selectively, but reversibly, inhibit metalloproteases.

Regional variations in the rate of tumour invasion have been observed in vivo. For example, prostatic malignancies often travel to the axial skeleton rather than invade locally (Bailey \& Love (1995)). These variations are thought to be due to variations in the composition of the ECM (Aznavoorian et al. (1990) and Liotta (1986)). In order to test this hypothesis in vitro, Perumpanani \& Byrne (1999) performed a series of invasion assays in which the density of the gel into which the tumour cells invaded was the key parameter. In more detail, HT1080 human fibrosarcoma cells were added to prepared collagen gels of different (but uniform) concentrations. HT1080 cells were used because they are known to respond to 


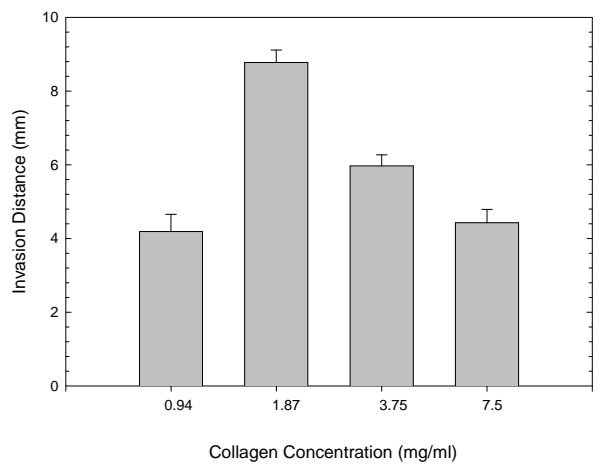

FIG. 1. Experimental results reproduced with permission from Perumpanani \& Byrne (1999) illustrating the biphasic relationship between tumour invasiveness and the concentration of collagen. Dose response curves of HT1080 invasion distance are shown for various concentrations of Type I collagen.

externally imposed haptotactic gradients but to undergo little chemokinetic motion (Aznavoorian et al. (1990)). In the experiments, no external gradients were imposed so any motion was due either to random motion or haptotaxis, the relevant growth factors being expressed by the HT1080 cells themselves.

When the invasiveness of the cells was measured, a biphasic relationship between the speed of the invading front and the concentration of the collagen gel was observed (see Figure 1). For small collagen concentrations the rate of invasion increased with the collagen concentration until it reached a maximum level. Thereafter the speed of invasion decreased with increasing collagen gel concentration. Perumpanani \& Byrne (1999) postulated that the biphasic relationship was due to interactions between cell proliferation and migration and developed a mathematical model to test this hypothesis. In addition to reproducing the observed biphasic behaviour, numerical simulations obtained from the mathematical model suggested that there was a biphasic relationship between collagen concentration and cell proliferation, a prediction that was then confirmed experimentally (see Figure 2).

As stated above, the model of tumour invasion presented by Perumpanani \& Byrne (1999) is based upon the three-step hypothesis. Other, complementary models have been developed to study the impact on invasion of other factors, including local pH levels (Gatenby \& Gawlinski (1996)), the mechanical properties of the gel or tissue in which the tumour is invading (Chen et al. (2001)), and differential proliferation rates of normal and cancer cells (Sherratt \& Nowak (1992)). By adapting a technique used previously to study angiogenesis, Anderson et al. (2000) developed a discrete model of tumour invasion and compared the results of model simulations with those from an equivalent, continuous and deterministic model. More recently Anderson (2005) has extended this discrete modelling approach by developing a hybrid mathematical model to study the impact of tumour cell heterogeneity on the invasion of vascular tumours (the model is termed hybrid since it combines continuous and discrete variables).

A weakness of many invasion models is that they are not amenable to analysis and must be solved using numerical methods. In consequence it is often difficult to assess the relative importance of the various mechanisms present in a model and how they interact. By contrast, Pettet et al. (2000) and others (Landman et al. (2003), Marchant et al. (2000) and Perumpanani et al. (1996)) have developed simple models for cell migration that are amenable to analysis in which chemotaxis is the dominant mechanism of cell migration. In particular, using non-standard phase place techniques, Marchant $e t$ al. 


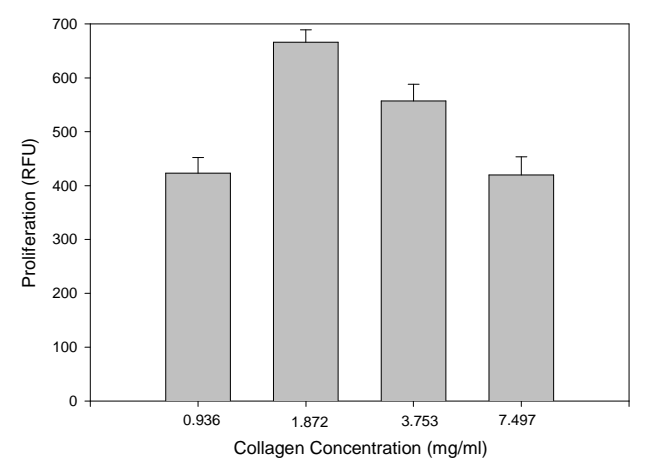

FIG. 2. Experimental results reproduced with permission from Perumpanani \& Byrne (1999) illustrating the biphasic relationship between tumour proliferation and the concentration of collagen. Dose response curves for HT1080 proliferation are shown for various concentrations of Type I collagen. Proliferation is measured in Reference Fluorescence Units (RFUs).

(2000) have demonstrated that such models may admit stable travelling shock wave solutions, in which the invading tumour profile and retreating collagen profiles are of fixed shape and move at a constant speed. The (stable) solutions are further characterised by a discontinuity, or blunt interface, at the front of the tumour wave, ahead of which the tumour concentration is zero (for a typical profile, see Figure 11) and, as such, may be deemed more physically realistic than the smooth-fronted solutions that arise when linear diffusive phenomena dominate cell migration (see, for example, the models developed by Anderson et al. (2000), Gatenby \& Gawlinski (1996) and Sherratt \& Nowak (1992)).

In view of the above observations, in this paper we develop a simple model of malignant invasion that retains the key features of the model presented by Perumpanani \& Byrne (1999) and, in particular, the biphasic dependence of the invasion speed on the density of the surrounding collagen gel but neglects random motion and diffusive effects. In this respect the model is similar in form to that analysed by Marchant et al. (2000). However an additional term is included here to represent the effect of competition for space between the collagen gel and the malignant cells. It is this term that ensures our model reproduces the biphasic behaviour observed in Perumpanani and Byrne's more complex model. Importantly, the model is also amenable to analysis using techniques similar to those developed by Pettet et al. (2000), Marchant et al. (2000) and Landman et al. (2003). Thus, we analyse our model within the framework of a nonstandard two-dimensional phase plane in which solutions may develop shocks or jump discontinuities. Discontinuous solutions may be constructed by connecting trajectories that pass through specific points on a singular curve in phase space and recross it via a shock when they intersect another, well-defined curve, termed the jump curve. When competition for space is omitted we recover the model developed by Marchant et al. (2000). In this case for each set of parameter values a unique, blunt interface travelling wave solution exists and therefore there is a monotonic relationship between the density of the surrounding collagen gel and the invasion speed. In this paper we show that the biphasic behaviour exhibited by our model is due to the inclusion of competition for space between the collagen gel and the malignant cells and may be explained using nonstandard phase plane analysis. For certain parameter values, it is possible to show that the competition mechanism leads to the existence of two distinct points at which trajectories may cross the singular curve and, hence, two distinct, discontinuous travelling wave solutions which lead directly to the biphasic behaviour. 
Working with a different model, Landman et al. (2003) have already recorded the possible existence of multiple points at which trajectories may cross the singular curve. However, to the best of our knowledge, this is the first time that the appearance of multiple points of this kind have been linked with nonuniqueness of travelling shock wave solutions. It is noteworthy that these shock solutions can be robustly stable (i.e. they persist as solutions of the full system of partial differential equations) and, hence, that they may describe what happens in vivo.

The remainder of the paper is organised as follows. In Section 2 we present our model of malignant invasion. Travelling shock wave solutions of our model are described in Section 3. Solutions of the model with and without competition for space between the connective tissue and the malignant cells are compared within a singular phase plane. This leads to a mathematical explanation of how competition for space results in a biphasic relationship between the farfield collagen concentration and the speed of invasion. Finally our conclusions are presented in Section 4.

\section{Model Derivation}

In this section we present our model of malignant invasion. As we explain below, it captures features of two existing models of invasion studied in Perumpanani et al. (1996) and Perumpanani \& Byrne (1999). For simplicity, we consider a one-dimensional Cartesian geometry. Denoting time and position by $t$ and $x$ and assuming that invasion takes place in the $x$-direction, we formulate our model in terms of three key dependent variables: $u(x, t)$, the concentration of tumour cells; $c(x, t)$, the concentration of connective tissue (which is formed of extracellular matrix elements); and $p(x, t)$, the concentration of a matrix-degrading protease that is produced by the tumour cells to facilitate their invasion.

The dominant mechanisms governing the evolution of the malignant cells are assumed to be proliferation and haptotaxis up spatial gradients of connective tissue. Following Perumpanani \& Byrne (1999), we assume that in the absence of connective tissue the cancer cells undergo logistic growth, with growth rate $r$ and carrying capacity $U_{0}$. The presence of extracellular matrix (ECM) leads to competition for space and a consequent reduction in the cancer cells' net proliferation rate. We model this effect by including a death term which is proportional to the product $u c$, with constant of proportionality $k$. Combining these ideas and denoting by $\chi$ the assumed constant haptotaxis coefficient, we deduce that

$$
\frac{\partial u}{\partial t}=\underbrace{r u\left(1-\frac{u}{U_{0}}\right)}_{\text {logistic growth }}-\underbrace{k u c}_{\begin{array}{c}
\text { competition } \\
\text { for space }
\end{array}}-\underbrace{\chi \frac{\partial}{\partial x}\left(u \frac{\partial c}{\partial x}\right)}_{\text {haptotaxis }} .
$$

We assume that, over the timescale of interest, ECM turnover may be neglected and that its evolution is dominated by protease degradation which takes place at a rate proportional to the product $c p$. Denoting by $\alpha$ the constant of proportionality, we deduce that

$$
\frac{\partial c}{\partial t}=-\alpha c p
$$

Finally, we assume that the invasive cells produce protease on contact with the ECM at a rate which is proportional to the product $u c$. The protease also undergoes natural decay. Combining these features we deduce that

$$
\frac{\partial p}{\partial t}=\beta u c-\gamma p
$$


In (2.3) the non-negative constants $\beta$ and $\gamma$ represent respectively the rate at which tumour cells produce protease and the natural decay rate of the protease.

Before introducing boundary and initial conditions that close equations (2.1)-(2.3), it is convenient to nondimensionalise our model equations and, thereby, derive the simplified model of malignant invasion that forms the basis for the analysis contained in this paper. We note that the major results of this work do not depend on many of the precise forms adopted above (for instance, the degradation term appearing in equation (2.2) could be replaced by the more general term $c p^{n}$ for $n$ an index near to 1); however, we use the above explicit forms to simplify the following presentation and to compute particular examples of solutions.

We denote by tildes dimensionless quantities and scale the independent and dependent variables as follows:

$$
t=T \tilde{t}, \quad x=L \tilde{x}, \quad u=U_{0} \tilde{u}, \quad c=C_{0} \tilde{c}, \quad p=P_{0} \tilde{p},
$$

with

$$
T=r^{-1}, L^{2}=\chi T C_{0}, C_{0}=\frac{\gamma P_{0}}{\beta U_{0}}, P_{0}=\frac{1}{\alpha T} .
$$

Under this transformation equations (2.1)-(2.3) become

$$
\begin{gathered}
\frac{\partial \tilde{u}}{\partial \tilde{t}}=\tilde{u}(1-\tilde{u}-\tilde{k} \tilde{c})-\frac{\partial}{\partial \tilde{x}}\left(\tilde{u} \frac{\partial \tilde{c}}{\partial \tilde{x}}\right), \\
\frac{\partial \tilde{c}}{\partial \tilde{t}}=-\tilde{c} \tilde{p}, \\
\frac{\partial \tilde{p}}{\partial \tilde{t}}=\frac{1}{\varepsilon}(\tilde{u} \tilde{c}-\tilde{p}),
\end{gathered}
$$

where

$$
\tilde{k}=\frac{k C_{0}}{r}, \quad \tilde{D}=\frac{D T}{L^{2}}, \quad \varepsilon=\frac{1}{\gamma T} .
$$

We remark that $t$ is scaled so that $u$ grows on an $O(1)$ timescale, $x$ is scaled so that the space and time scales for haptotaxis are also $O(1), p$ is scaled so that $c$ is degraded on the same timescale and, finally, $c$ is scaled so that the dimensionless rates of protease production and decay in equation (2.6) are identical.

In equation (2.6), the constant $\varepsilon$ relates the timescale associated with the protease dynamics to that of the tumour cell dynamics. Following Perumpanani et al. (1996) we exploit the small size of $\varepsilon$ to write $\tilde{p}=\tilde{u} \tilde{c}+\mathscr{O}(\varepsilon)$ in place of (2.6) (this approximation is appropriate provided that $\partial \tilde{p} / \partial \tilde{t}$ is bounded). Omitting tildes henceforth, in the limit as $\varepsilon \rightarrow 0$ we set $p=u c$ and obtain the following system of equations for $u$ and $c$ :

$$
\begin{aligned}
\frac{\partial u}{\partial t} & =u(1-u-k c)-\frac{\partial}{\partial x}\left(u \frac{\partial c}{\partial x}\right) \\
\frac{\partial c}{\partial t} & =-u c^{2}
\end{aligned}
$$

A novel feature of our model that distinguishes it from existing models (Orme \& Chaplain (1996), Perumpanani \& Byrne (1999) and Anderson et al. (2000)) is the absence of diffusion. This is reasonable for the ECM since it is a large immobile matrix. In addition, malignant cell lines and proteases that exhibit minimal diffusion have been observed. Aznavoorian et al. (1990) saw that the haptotactic 

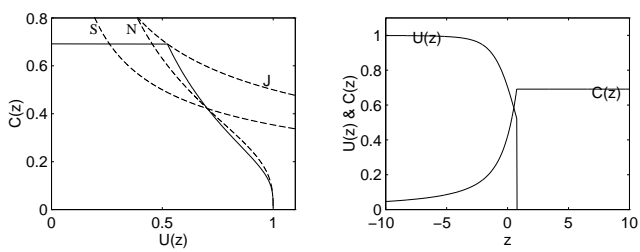

FIG. 3. A phase plane connection and a solution profile for the blunt interface travelling wave solution of (2.7)-(2.8) for $a=0.5$ and $k=0$. In the solution profiles, the blunt interface in the $U$ profile can be clearly seen as can the corresponding corner in the $C$ profile.

response of the HT1080 melanoma cells studied in Perumpanani \& Byrne (1999) was at least fifty times greater than the random response. Furthermore, Tsuboi \& Rifkin (1990) stated that uPA, a protease produced by HT1080 cells, is bound to the ECM and therefore does not diffuse.

We show below that our reduced model exhibits the same biphasic behaviour as the more complex invasion model developed in Perumpanani \& Byrne (1999). Analytical and numerical studies described in Marchant et al. (2001) suggest that the inclusion of a small amount of tumour cell random motion doesn't affect the qualitative behaviour of the model equations. In this respect we believe that our model captures the key features of tumour invasion needed to realise the observed nonlinear dependence of the tumour invasion speed on the density of the surrounding material, which will be proportional to the local value of $c$.

We now motivate the boundary and initial conditions that we use to close equations (2.7)-(2.8). We are considering the invasion of malignant cells, initially located near (and to the left of) $x=0$ in a neighbourhood devoid of ECM. The farfield (in the $x>0$ direction) is devoid of tumour cells and occupied by ECM at some positive concentration $\hat{c}$, say. Thus we have initially

$$
u(x, 0)=u_{0}(x) \text { and } c(x, 0)=c_{0}(x)
$$

while as time increases

$$
u(0, t)=1,
$$

where the initial distributions $u_{0}(x)$ and $c_{0}(x)$ are such that

$$
\begin{gathered}
u_{0}(x)=1 \quad \text { and } \quad c_{0}(x)=0 \quad \text { at } x=0, \\
u_{0}(x) \rightarrow 0 \quad \text { and } \quad c_{0}(x) \rightarrow \hat{c} \quad \text { as } x \rightarrow \infty .
\end{gathered}
$$

We note that our model comprises equations (2.7)-(2.10) and contains only two parameters: $k$ and $\hat{c}$, where $k$ is the relative strength of the crowding effect from $c$ to that from itself, and $\hat{c}$ is the scaled level of connective tissue (ECM) in the absence of malignant cells.

\section{Travelling Wave Solutions}

Marchant et al. (2000) studied travelling wave solutions of (2.7)-(2.8) in the absence of contact inhibition. In particular, using singular phase plane analysis, they demonstrated the existence of a family of discontinuous travelling wave solutions to (2.7)-(2.8) for $k=0$. Furthermore numerical simulations demonstrated that a member of this family of solutions with a blunt interface at $u(x, t)=0$ evolved stably 
from biologically-realistic, compact initial data. One such solution existed for each set of parameters leading to a monotonic relationship between the far-field, tumour-free tissue concentration, $\hat{c}$ and the invasion speed, $a$. Figure 3 shows the travelling wave phase plane trajectory and the solution profile for such a blunt interface travelling shock wave occurring when $k=0$. Recall that when contact inhibition was included by Perumpanani \& Byrne (1999) a biphasic relationship between these quantities was observed (see Figure 1). Therefore we now investigate the effect of having $k>0$ in (2.7)-(2.8) and in particular how, for travelling wave solutions, the relationship between $\hat{c}$ and $a$ changes when $k>0$.

We look for constant form travelling wave solutions for which

$$
u(x, t) \equiv U(z), c(x, t) \equiv C(z), z \equiv x-a t,
$$

where $z$ is the travelling wave coordinate and the wavespeed $a$ is an unknown (eigenvalue) which we determine as part of the solution. Under this transformation equations (2.7)-(2.8) reduce to the following third order system of ordinary differential equations (ODEs):

$$
\begin{aligned}
-a \frac{d U}{d z} & =U(1-U-k C)-\frac{d}{d z}\left(U \frac{d C}{d z}\right), \\
a \frac{d C}{d z} & =U C^{2} .
\end{aligned}
$$

while equations (2.9)-(2.10) supply

$$
\begin{gathered}
U \rightarrow 1, \quad C \rightarrow 0 \quad \text { as } z \rightarrow-\infty \\
U \rightarrow 0, \quad C \rightarrow \hat{c} \quad \text { as } z \rightarrow \infty
\end{gathered}
$$

where $\hat{c}>0$. These limits are steady states of equations (3.1)-(3.2): $(U, C)=(1,0)$ represents a situation where only malignant cells are present and $(U, C)=(0, \hat{c})$ a situation where only healthy tissue is present. Thus we seek a heteroclinic connection between the malignant and normal steady state solutions.

Using (3.2) we substitute for $d C / d z$ in (3.1), thereby reducing the system to the following nonstandard, second-order form:

$$
\begin{aligned}
\left(\frac{2 U C^{2}}{a}-a\right) \frac{d U}{d z} & =U\left(1-U-k C-\frac{2 U^{2} C^{3}}{a^{2}}\right), \\
\frac{d C}{d z} & =\frac{U C^{2}}{a} .
\end{aligned}
$$

We solve (3.5)-(3.6) subject to (3.3)-(3.4). Henceforth, it is convenient to regard $a>0$ as fixed and $\hat{c}$ as an unknown eigenvalue.

The parameter $k$ only appears once in (3.5)-(3.6). Therefore there are similarities between this system and the $k=0$ system studied in Marchant et al. (2000). In particular equation (3.5) becomes singular on the curve

$$
\mathrm{S}: 2 U C^{2}=a^{2},
$$

which we term the singular barrier. Phase plane trajectories have no meaning on S and can only cross it when the $U$ nullcline,

$$
\mathrm{N}: U(1-U-k C)-\frac{2 U^{3} C^{3}}{a^{2}}=0
$$


intersects $\mathrm{S}$, since at this point $d U / d z$ may still be finite (because of the zero/zero indeterminate form). Following Pettet et al. (2000), we call such points "holes in the wall", H.

Figures 3 and 4 illustrate how the curve $N$ varies with $k$. Whereas $C \rightarrow \infty$ as $U \rightarrow 0$ when $k=0$ (see Figure 3), when $k>0$ the curve $\mathrm{N}$ crosses $U=0$ at $C=1 / k$ (see Figure 4). This does not affect the nature of the malignant steady state $(U, C)=(1,0)$ (it still has an unstable manifold and an unstable centre manifold), although it does affect the nature of the healthy steady states $(U, C)=(0, \hat{c})$ : for $\hat{c}>1 / k$ they are unstable whereas for $\hat{c}<1 / k$ they remain (linearly) stable. The inclusion of contact inhibition also alters the number and positions of the holes in the wall. When $k=0$, one hole in the wall exists where $\mathrm{S}$ and $\mathrm{N}$ intersect. By substituting $2 U C^{2}=a^{2}$ into (3.8) for $k>0$ we deduce that at a hole in the wall $C=C_{H}$ where

$$
2 k C_{H}^{3}+a^{2} C_{H}+a^{2}-2 C_{H}^{2}=0 .
$$

For example, if $a \ll 1$ and $k=O(1)$ then there are two physically relevant holes in the wall (i.e. those in the positive quadrant), with coordinates $\left(U_{H}, C_{H}\right) \sim(1, a / \sqrt{2})$ and $\left(U_{H}, C_{H}\right) \sim\left(2 a^{2} k^{2}, 1 / 2 k\right)$.

If $k=0$ equation (3.9) has one positive real root and one negative real root. For $k>0$, using Descartes' Rule of Signs (Abramowitz \& Stegun (1965)) it is possible to show that two real positive roots exist if

$$
0<k<k^{*}=\frac{1}{27 a}\left(\left(6+a^{2}\right)^{3 / 2}-a^{3}-9 a\right) .
$$

In Figure 5 we plot the positive roots of (3.9) as $k$ varies, for $a>0$ fixed. This sketch shows how the number of holes in the wall varies with $k$ and suggests that the qualitative behaviour of our twodimensional phase plane depends crucially on the value of $k$. In particular for $k>k^{*}$ there are no holes in the wall.

Another similarity between the $k>0$ and $k=0$ models is that they admit the same hyperbolic jumps since $k$ does not appear in any of the derivative terms. Marchant et al. (2000) showed that hyperbolic jumps which satisfied the Lax entropy condition (Courant \& Hilbert (1962)) and were therefore stable to added viscosity, could take a trajectory directly from the curve (where $U=U_{L}, C=C_{L}$ )

$$
J: U C^{2}=a^{2}
$$

to $(U, C)=(0, \hat{c})=\left(U_{R}, C_{R}\right)$ and that $C$ remains fixed over this jump (i.e. $\left.C_{L}=C_{R}=\hat{c}\right)$. Here $U_{L}, C_{L}$, $U_{R}$ and $C_{R}$ denote the values of $U$ and $C$ on the left and right of the jump. Thus, valid travelling wave solutions may involve a smooth connection between $(1,0)$ and the curve $\mathbf{J}$, with a jump from $\mathbf{J}$ to $(0, \hat{c})$ completing the heteroclinic connection.

Marchant et al. (2000) observed from numerical solutions of the $k=0$ case that the solutions which evolved from general initial data included a jump from the curve $\mathrm{J}$ to a normal steady state $(U, C)=$ $(0, \hat{c})$. We note that the mid-point of a jump from $\mathrm{J}$ to $(0, \hat{c})$ lies on the singular barrier $\mathrm{S}$, and that the two steady states $(1,0)$ and $(0, \hat{c})$ lie on the opposite side of $\mathrm{S}$ to the curve $\mathrm{J}$. A connection between $(1,0)$ and $\mathrm{J}$ must cross $\mathrm{S}$ at a hole in the wall, $\mathrm{H}$. Thus, in order to determine whether a travelling wave of the desired form can occur one must determine whether a trajectory that crosses $\mathrm{S}$ at $\mathrm{H}$ eventually intersects J. Marchant et al. (2000) showed that when $k=0$ and for all values of $a$, a trajectory exists which leaves the malignant steady state $(U, C)=(1,0)$, crosses the singular barrier $\mathrm{S}$ at $\mathrm{H}$ and then crosses $\mathrm{J}$. An example of such a trajectory is shown in Figure 3. We now investigate whether such trajectories exist when $k>0$. We have described how holes in the wall only exist for $k<k^{*}$. Thus, for each value of $a$, there is an upper bound on the values of $k$ for which blunt travelling shock waves exist. This critical value of $k=k^{*}$ decreases monotonically with $a$ according to (3.10). 


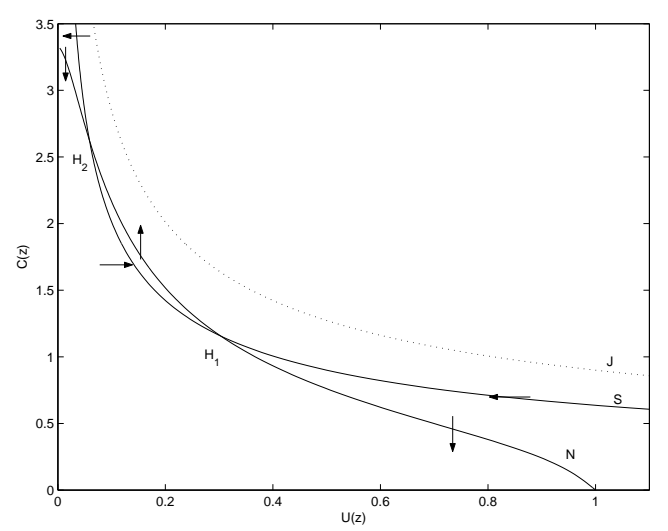

FIG. 4. The $U$ and $C$ nullclines and the jump curve, denoted $\mathrm{N}, \mathrm{S}$ and $\mathrm{J}$ respectively, of the regularised system when $k=0.3<k^{*}$ and $a=0.9$. The $C$ nullcline coincides with the singular barrier $\mathrm{S}$ of the original, singular system. The arrows indicate the vector field directions across the nullclines. Note the effect of $k$ in the top left hand corner of the diagram.

\subsection{Regularising the Phase Plane}

In this section we assume $0<k<k^{*}$ and investigate how competition for space affects the existence and uniqueness of heteroclinic connections between the malignant and normal steady state solutions for fixed values of $a$. When $0<k<k^{*}$ the $U$ nullcline and singular barrier intersect twice in the positive quadrant of the phase plane (see Figure 4). We denote by $\mathrm{H}_{1}$ the point of intersection with the lowest value of $C_{H}$ and remark that it corresponds to the hole in the wall of the $k=0$ problem. The other intersection, $\mathrm{H}_{2}$, occurs due to the altered behaviour of the $U$ nullcline when $0<k<k^{*}$.

To understand the behaviour close to $\mathrm{H}_{1}$ and $\mathrm{H}_{2}$ we regularise equations (3.1)-(3.2) via the travelling wave variable $Z$ defined by $d / d Z=\left(2 U C^{2} / a-a\right) d / d z$ to obtain

$$
\begin{aligned}
& \frac{d U}{d Z}=U(1-U-k C)-\frac{2 U^{3} C^{3}}{a^{2}} \\
& \frac{d C}{d Z}=U C^{2}\left(\frac{2 U C^{2}}{a^{2}}-1\right) .
\end{aligned}
$$

In this regularised system, the singular barrier $S$ is transformed to a $C$ nullcline, the direction of trajectories to the left of $\mathrm{S}$ (i.e. when $2 U C^{2}<a^{2}$ ) is reversed and $\mathrm{H}_{1}$ and $\mathrm{H}_{2}$ are steady states.

In Figure 4 we plot the nullclines $\mathrm{N}$ and $\mathrm{S}$ of the transformed system with the jump curve $\mathrm{J}$ for typical values of $a$ and $k$. The direction of the vector field across these nullclines is marked so that the nature of the steady states $\mathrm{H}_{1}$ (a saddle) and $\mathrm{H}_{2}$ (a stable spiral) is evident.

As for $k=0$ (Marchant et al. (2000)), a connection exists between the unstable manifold of $\mathrm{H}_{1}$ and the malignant steady state $(U, C)=(1,0)$. The problem of finding travelling shock waves with a blunt interface reduces to following this trajectory on the other side of $S$ and seeing whether it crosses the curve J. In the regularised system trajectories leaving $\mathrm{H}_{1}$ eventually spiral to $\mathrm{H}_{2}$ (see Figure 7) and, in so doing, they cross S. In the original, singular system a trajectory is unable to cross the singular barrier (except at holes in the wall). Therefore in the regularised plane we only need follow the trajectory until the point $P_{\text {end }}$ at which it hits $\mathrm{S}$. Since both $\mathrm{H}_{1}$ and $P_{\text {end }}$ lie to the left of the curve $\mathrm{J}$, in moving between $\mathrm{H}_{1}$ and $P_{\text {end }}$ the orbit necessarily crosses $\mathrm{J}$ an even number of times (either 0 or 2 ), each intersection giving rise to a different, blunt-form travelling wave solution, for particular values of $a$ and $k$. 


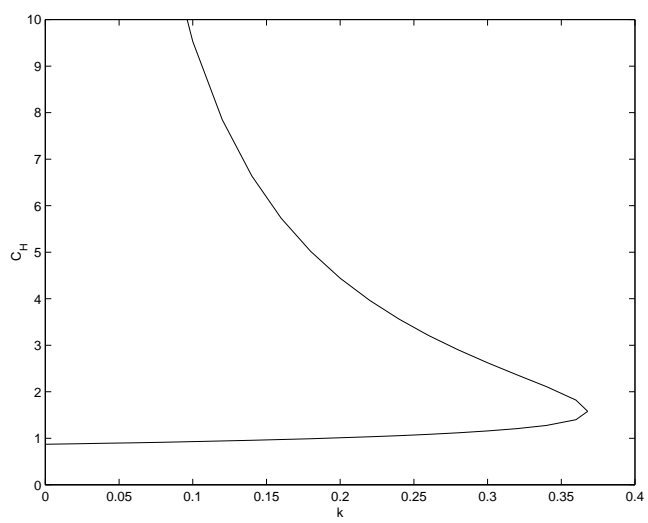

FIG. 5. A sketch showing how the positive solutions $C_{H}$ of (3.9) depend upon $k$ for a fixed value of $a$. Parameter values: $a=0.9, k^{*}=0.37$.

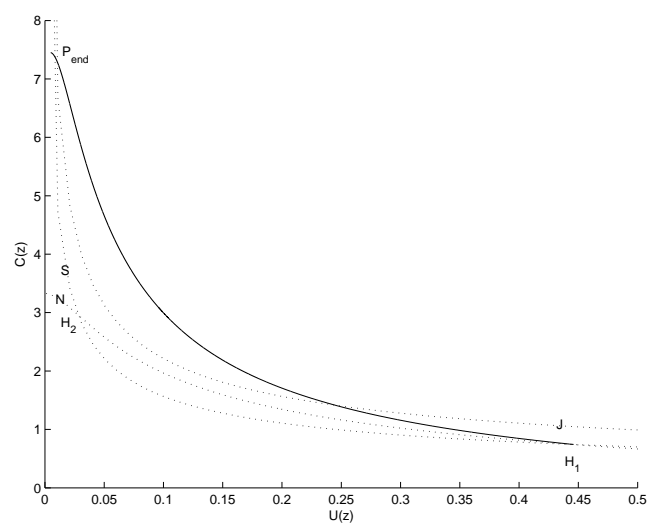

FIG. 6. A plot of a trajectory (the solid line) leaving the steady state $\mathrm{H}_{1}$ of the regularised system when $k=0.3$ and $a=0.7$. The trajectory intersects the $\mathrm{J}$ curve twice; first when $C=1.415$ and second when $C=7.290$.

We illustrate in Figures 6 and 7 how varying $a$ affects the existence of such travelling wave solutions. Figure 6 shows the regularised phase plane trajectory which leaves $\mathrm{H}_{1}$ and moves into the region to the right of $\mathrm{S}$ for $a=0.7$ and $k=0.3$. This trajectory crosses the $\mathrm{J}$ curve twice, the first intersection occurring when $C=1.415$ and the second when $C=7.290$. Hence we anticipate that when $k=0.3$, two blunt-form travelling wave solutions exist with wavespeed $a=0.7$. One of these solutions has $\hat{c}=1.415$ and the other $\hat{c}=7.290$. In Figure 7 the trajectory leaving $\mathrm{H}_{1}$ is plotted for $k=0.3$ and $a=0.9$. In this case no intersections between the trajectory and the $\mathrm{J}$ curve occur so we conclude that no blunt form travelling wave solutions exist for these values of $a$ and $k$.

We may explore how the number of blunt form solutions varies with $k$ via a continuity argument. We note that the $\mathrm{S}$ and $\mathrm{J}$ curves are independent of $k$. For a fixed value of $C$, the $U$ ordinate of the $U$ nullcline $\mathrm{N}$ decreases as $k$ increases. Therefore $\mathrm{H}_{1}$ moves along $\mathrm{S}$, in the direction of decreasing $U$, as $k$ is increased. The regularised travelling wave system (3.12)-(3.13), or the orbits satisfying (3.5)-(3.6), 


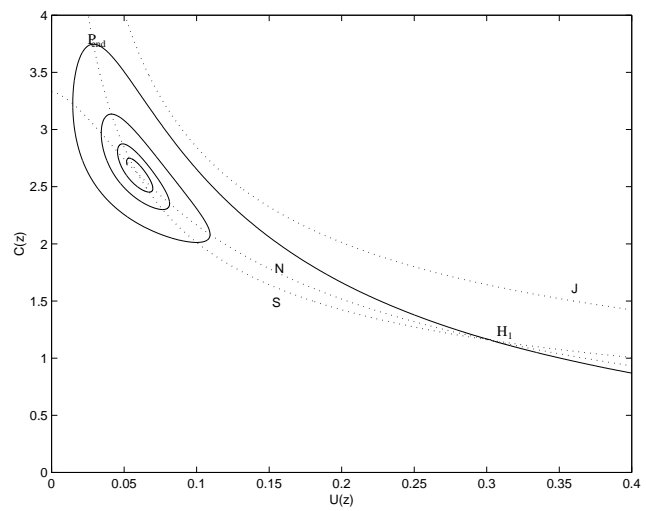

FIG. 7. A plot of a trajectory (the solid line) leaving the steady state $\mathrm{H}_{1}$ of the regularised system when $k=0.3$ and $a=0.9$. The curves $\mathrm{J}$ and $\mathrm{S}$ are unchanged, whereas $\mathrm{N}$ is changed, especially near the $C$ axis.

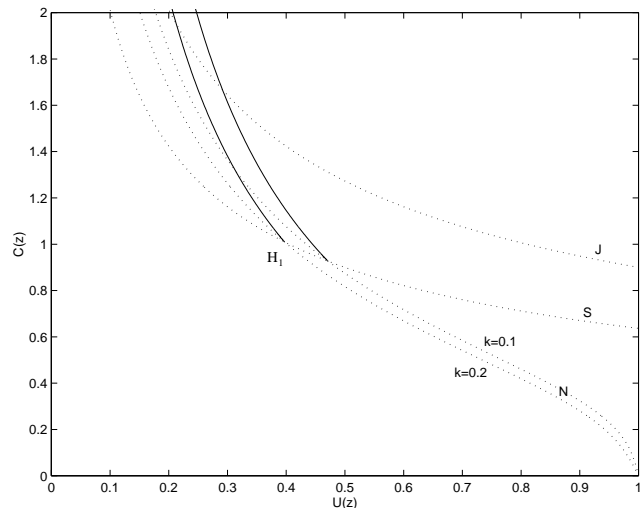

FIG. 8. An illustration of the continuity argument which demonstrates that $\hat{c}_{\max }$ increases with $k$. The singular barrier/C nullcline and $\mathrm{J}$ curve for $a=0.9$ are shown along with the corresponding $U$ nullclines for $k=0.1,0.2$. The hole in the wall $\mathrm{H}_{1}$ moves from right to left as $k$ is increased. The trajectories leaving $\mathrm{H}_{1}$ are also marked. $d C / d U$ becomes less negative as $k$ is increased and thus the $C$ value where the trajectories cross $\mathrm{J}$ increases.

may be written in the form

$$
\frac{d U}{d C}=\frac{a^{2}(1-U-k C)-2 U^{2} C^{3}}{C^{2}\left(2 U C^{2}-a^{2}\right)} .
$$

When a trajectory originating from $(U, C)=(1,0)$ crosses $\mathrm{H}_{1}$ it enters a region of the phase plane where $d U / d C$ is negative. When $k=0$, the trajectory intersects $\mathrm{J}$ once. As $k$ increases, $d C / d U$ becomes less negative and, by continuity, the $C$-coordinate of the point at which the trajectory first intersects $\mathrm{J}$ increases with $k$. We refer to this value as $\hat{c}_{1}$. We know that the trajectory intersects $\mathbf{J}$ either not at all or twice. Therefore, if the trajectory intersects once there must be a second intersection at $C=\hat{c}_{2}$. Continuity requires that $\hat{c}_{2}$ decreases for increasing $k$.

Thus for small values of $k$, intersections occur at $C=\hat{c}_{1}$ and $C=\hat{c}_{2} \sim O(1 / k)$ and there are two values of $\hat{c}$ for which blunt-form travelling wave solutions exist. As $k$ is increased eventually a threshold value $k=k_{\max }<k^{*}$ is reached at which $\hat{c}_{1}=\hat{c}_{2}$ and the trajectory makes a tangent with $\mathrm{J}$ at the 
intersection point. At this intersection

$$
\left.\frac{d U}{d C}\right|_{\text {trajectory }}=\left.\frac{d U}{d C}\right|_{J} .
$$

Combining (3.14) and (3.11) yields that

$$
\left.\frac{d U}{d C}\right|_{\text {trajectory }}=\frac{C^{2}-a^{2}-k C^{3}-2 a^{2} C}{C^{4}}
$$

and

$$
\left.\frac{d U}{d C}\right|_{J}=\frac{-2 a^{2}}{C^{3}}
$$

Therefore when the trajectory intersects $\mathrm{J}$ at a tangent,

$$
k=k_{\max }=\frac{1}{C}\left(1-\frac{a^{2}}{C^{2}}\right),
$$

when the trajectory crosses $\mathrm{J}$ for the first time

$$
k>\frac{1}{C}\left(1-\frac{a^{2}}{C^{2}}\right)
$$

and when it crosses for the second time

$$
k<\frac{1}{C}\left(1-\frac{a^{2}}{C^{2}}\right) .
$$

No intersections between the trajectory and $\mathbf{J}$ (and hence no travelling waves with compact support) exist when $k>k_{\max }$.

\subsubsection{Asymptotic Behaviour}

It is possible to gain further insight into the differences in behaviour between the cases $k=0$ and $0<k \ll 1$ by constructing approximate solutions of equation (3.14) for large $C$. Numerical solutions of (3.14) (see Figure 6) demonstrate that the phase plane trajectories for the two cases are qualitatively similar for small $C$. They both smoothly connect $(U, C)=(1,0)$ to $\mathrm{H}_{1}$ and then continue on the other side of the singular barrier. It is only when $C$ becomes large that the effect of the $-k U C$ term becomes apparent. When $k=0$ the trajectory remains to the right of $\mathrm{N}$ and $\mathrm{S}$ for all further $Z$ but when $k>0$ the gradient $d C / d U$ increases (becomes less negative) and eventually becomes zero as it turns over and hits the singular barrier. It is this behaviour which allows the trajectory to cross $\mathrm{J}$ twice. We consider this behaviour below by constructing an asymptotic solution starting after the trajectory has moved away from $\mathrm{H}_{1}$.

We study equation (3.14) in a region where $C$ and $2 U C^{2}$ are large and $U$ is small. We assume $U \ll 1$ and $a^{2} \ll U C^{2}$, and introduce a new variable, $w_{1}=U C^{3 / 2} / a$ which we substitute into (3.14) to obtain:

$$
\frac{d\left(w_{1}^{2}\right)}{d(\ln C)}=w_{1}^{2}+1-k C \text {. }
$$


On setting $w_{2}=w_{1}^{2}$ and $\zeta=\ln C,(3.16)$ may be re-written as

$$
\frac{d w_{2}}{d \zeta}=w_{2}+1-k \exp (\zeta)
$$

which has solution

$$
w_{2}(\zeta)=A \exp (\zeta)-1-k \zeta \exp (\zeta)
$$

for some constant $A$. In terms of the original variables we have

$$
\frac{U^{2} C^{3}}{a^{2}}=A C-1-k C \ln C .
$$

Therefore when $k=0, U C$ is approximately constant for large $C$ and $U C^{2}$ is increasing, so the trajectory may only cross $\mathrm{J}: U=a^{2} / C^{2}$ once. When $k>0$ and $C$ is large, the $-k C \ln C$ term eventually dominates causing the trajectory to bend towards $\mathrm{S}$ and cross $\mathrm{J}$ for a second time. This happens when

$$
C \approx \exp \left(\frac{A}{k}\right) .
$$

Thus we have characterised the behaviour of the phase plane trajectories when $C$ is large up to a constant parameter, $A$. In order to determine $A$ analytically it would be necessary to construct an asymptotic approximation that is valid for the entire trajectory, determining $A$ by matching across the different regions of the phase plane. A simple way to see this is to fix $C=1$ or $C=C_{0}$ as the starting point of this curve, with corresponding value $U=U_{0}$. Then, for $k$ small, we have $U_{0}^{2} C_{0}^{3}=a^{2}\left(A C_{0}-1\right)$, and this fixes $A$. Hence we need to find the $U_{0}$-value for curves leaving the hole in the wall when $0<a \ll 1$. We could use numerical methods to find $A$; the difficulty is that the appearance of $\log C \operatorname{loses}$ accuracy significantly.

\subsection{Solutions to the PDE model for $k>0$}

Our analysis in the travelling wave phase plane has shown that for each particular combination of $k$ and $a$ there exists either zero, one or two blunt interface travelling shock wave solutions to (2.7)-(2.8). In the phase plane we fix $a$ and $k$ and determine values of $\hat{c}$ that give rise to blunt interface solutions. By contrast, when solving the PDE system we fix $\hat{c}$ and $k$ and calculate the resulting invasion speed $a$.

Having demonstrated the existence of travelling shock wave solutions to (2.7)-(2.8) when $k>0$ we now investigate whether such solutions are biologically relevant via numerical simulations. Following Marchant et al. (2000) we solve equations (2.7)-(2.8) for $k>0$ with a Lax Friedrichs numerical scheme. The Lax Friedrichs method approximates conservation laws of the form

$$
\mathbf{u}_{t}+(\mathbf{f}(\mathbf{u}))_{x}=\mathbf{g}(\mathbf{u}),
$$

for vector functions $\mathbf{f}$ and $\mathbf{g}$ of vector variable $\mathbf{u}$ by the scheme

$$
\mathbf{u}_{j}^{n+1}=\frac{1}{2}\left(\mathbf{u}_{j-1}^{n}+\mathbf{u}_{j+1}^{n}\right)-\frac{\Delta t}{2 \Delta x}\left(\mathbf{f}\left(\mathbf{u}_{j+1}^{n}\right)-\mathbf{f}\left(\mathbf{u}_{j-1}^{n}\right)\right)+\mathbf{g}\left(\mathbf{u}_{j}^{n}\right) .
$$

Here, $\mathbf{u}_{j}^{n}$ denotes the value of vector $\mathbf{u}$ at location $j$ and time $n$ where the location $x$ and time $t$ axes have been discretized with spacing $\Delta x$ and $\Delta t$ respectively. The Lax Friedrichs scheme is only first order accurate but it is simple to implement and is not prone to numerical artefacts. For these reasons we 


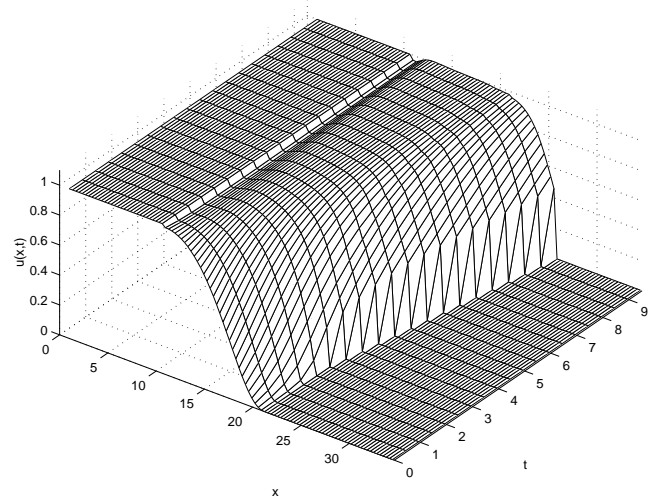

FIG. 9. A plot showing the evolution of the $u(x, t)$ profile of a Lax Friedrichs solution to (2.7)-(2.8) from smooth semi-compact initial data of the form (3.21)-(3.22) for $k=0$ and $\hat{c}=1.0$.

favour it over second order accurate schemes described in Leveque (1982) and Hundsdorfer \& Verwer (2003).

We assume realistic initial conditions for which the tumour cells have compact support. Typical results for the $k=0$ case presented in Figure 9 show the evolution of the $u(x, t)$ profile for initial data

$$
\begin{aligned}
& u_{0}(x)= \begin{cases}1, & \text { if } x<10, \\
\frac{1}{2} \cos \left(\frac{\pi(x-10)}{10}\right)+\frac{1}{2}, & \text { if } 10 \leqslant x \leqslant 20, \\
0, & \text { if } x>20,\end{cases} \\
& c_{0}(x)=\hat{c}\left(1-u_{0}(x)\right),
\end{aligned}
$$

with $\hat{c}=1.0$. Initially the interface between $u(x, t)>0$ and $u(x, t)=0$ remains stationary and the gradient of $u$ behind this interface increases. After a finite waiting time, $u$ develops a shock at the interface and this moves forwards at constant speed (waiting times are a characteristic feature of nonlinear diffusion problems, see, for example, Lacey et al. (1982)). For $k=0$ the wavespeed of solutions that evolve from initial data (3.21)-(3.22) increases with $\hat{c}$.

When $k>0$, blunt interface travelling wave solutions evolved from initial data of the form (3.21)(3.22). Figure 10 shows that for $k=0.3$ the relationship between the wavespeed $a$ of these solutions and the chosen farfield tissue concentration $\hat{c}$ is biphasic. This biphasic relationship may be inferred from the travelling wave phase plane analysis. When the PDEs (2.7)-(2.8) are solved for a small value of $\hat{c}$ then the resulting solution corresponds to a phase plane trajectory which jumps to $U=0$ at the first intersection with the $\mathrm{J}$ curve. For example, in Figure 11 we present travelling wave solution profiles for the case $k=0.3$ and $\hat{c}=1.415$. From Figure 6 we note that this is the lower value of $\hat{c}$ that occurs when $k=0.3$ and $a=0.7$. We remark further that the wavespeed predicted from the PDE system is in good agreement with that used to construct the travelling wave equations ( $a=0.695$ for the PDE system and $a=0.70$ for the travelling wave solution profiles). We note also that in both cases $k<\left(1-a^{2} / c^{2}\right) / c$ as predicted by the travelling wave analysis of Section 3.1 .

If $\hat{c}$ is increased then $a$ increases as when $k=0$ until a maximum value of $a$ is reached. This solution corresponds to a phase plane trajectory that makes a tangent with the $\mathrm{J}$ curve and on which $k=\left(1-a^{2} / c^{2}\right) / c$.

When $\hat{c}$ is increased further the wavespeed decreases. These solutions correspond to trajectories that 


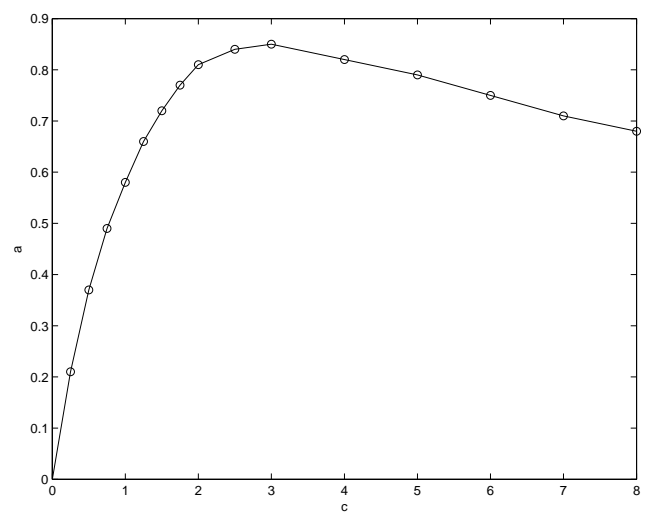

FIG. 10. A plot of the wavespeed $a$ of travelling wave solutions to (2.7)-(2.8) (evolving from semi-compact initial conditions) against the far-field concentration $\hat{c}$ of connective tissue when $k=0.3$. These wavespeeds were approximated from Lax Friedrichs numerical solutions to (2.7)-(2.8).

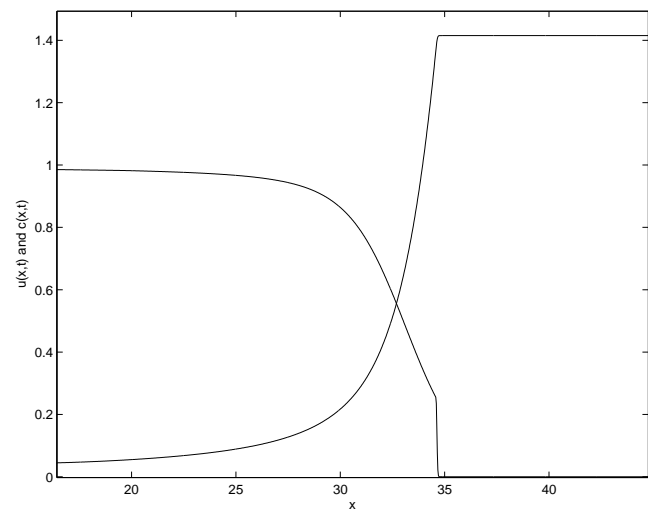

FIG. 11. Travelling wave profiles of $u(x, t)$ and $c(x, t)$ resulting from numerical solution of (2.7)-(2.8) with compact initial data (3.21)-(3.22) for $\mathrm{k}=0.3$ and $\hat{c}=1.415$. The measured wavespeed of the solution profiles was $a=0.695$.

jump to $U=0$ from the second intersection with the $\mathrm{J}$ curve. For example, Figure 12 shows travelling wave solution profiles for $k=0.3$ and $\hat{c}=7.290$, the higher value of $\hat{c}$ for which $k=0.3$ and $a=0.7$ (see Figure 6). Once again there was good agreement between the wavespeed calculated from the PDE system and that used for the travelling wave solution profiles $(a=0.7$ versus $a=0.702)$ and in both cases $k>\left(1-a^{2} / c^{2}\right) / c$.

We have shown that biologically relevant solutions of (2.7)-(2.8) are travelling shock waves and that they evolve stably from semi-compact initial data. Furthermore the biphasic relationship between the wavespeed of these solutions and the farfield tissue concentration may be explained within a singular phase plane. The wavespeed which results from solving (2.7)-(2.8) for particular values of $\hat{c}$ and $k$ may be determined by finding the value of $a$ for which a trajectory crossing the singular barrier of system (3.1)-(3.2) crosses the J curve at $c=\hat{c}$. Relationship (3.15) may be used to determine whether a particular solution lies to the left or the right of the maximum wavespeed in Figure 10 and whether increasing the amount of tissue present will increase or decrease the invasion speed. 


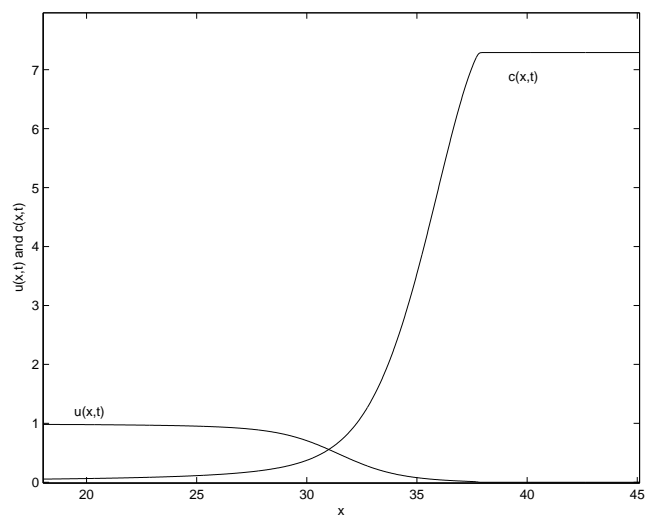

FIG. 12. Travelling wave profiles of $u(x, t)$ and $c(x, t)$ resulting from numerical solution of (2.7)-(2.8) with compact initial data (3.21)-(3.22) for $\mathrm{k}=0.3$ and $\hat{c}=7.290$. The measured wavespeed of the solution profiles was $a=0.702$.

\section{Conclusions and Discussion}

In this paper we have developed a simple, one-dimensional model of malignant invasion that combines features of two existing invasion models (see Perumpanani \& Byrne (1999) and Marchant et al. (2000)). For certain parameter values, the model admits discontinuous solutions for which the speed of invasion exhibits a biphasic dependence on the concentration of the surrounding gel or tissue, a characteristic feature of tumour invasion (see Figures 1 and 10). We are able to explain how this relationship arises by seeking steady travelling wave solutions to the governing equations and analysing the resulting system of ordinary differential equations within a singular, two-dimensional phase plane (Marchant et al. (2000)). The physically relevant (stable), discontinuous solutions are constructed by connecting trajectories that pass through specific points on a singular curve in phase space and recross it via a shock when they intersect the jump curve. Within this framework, the biphasic behaviour exhibited by our model was due to the inclusion of competition for space between the collagen gel and the malignant cells: for certain parameter values, this mechanism leads to the existence of two distinct points at which trajectories may cross the singular curve and, hence, two distinct, discontinuous travelling wave solutions. While Landman et al. (2003) have studied models which admit two "holes in the wall", to our knowledge, this is the first time that their existence has been related to that of pairs of travelling shock wave solutions which are stable in the sense that, where they exist, both are robust solutions of the underlying PDE model. Further work is needed to analyse the local and global stability of these discontinuous travelling wave solutions. Of additional interest would be investigating whether this result generalises to cases in which the model possesses three or more holes in the wall and whether other physical mechanisms can give rise to the same phenomenon. In order to address such questions, we propose to use similar phase plane techniques to analyse the following model

$$
\begin{gathered}
\frac{\partial u}{\partial t}=f(u, c)-\frac{\partial}{\partial x}\left(u \chi(c) \frac{\partial c}{\partial x}\right), \\
\frac{\partial c}{\partial t}=g(u, c),
\end{gathered}
$$

for different choices of the production terms $f(u, c), g(u, c)$ and the haptotaxis coefficient $\chi(c)$. 


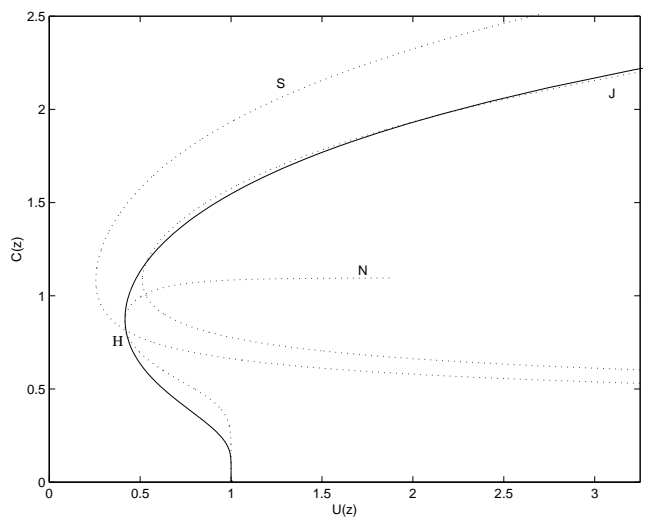

FIG. 13. A plot of the phase plane behaviour for (4.1)-(4.2) with $\chi(C)$ defined by (4.4) and $a=0.365$. The singular barrier $\mathrm{S}$ and the $U$ nullcline $\mathrm{N}$ intersect once at the hole in the wall $H$. The singular barrier lies between the malignant steady state $(U, C)=$ $(1,0)$ and the curve $\mathrm{J}$. The plot shows a trajectory leaving $(U, C)=(1,0)$, crossing the singular barrier at $\mathrm{H}$ and intersecting the curve $\mathrm{J}$ twice. From each intersections with $\mathrm{J}$ the trajectory may complete the travelling wave solution by jumping down to $U=0$, with a blunt wave-front.

Following the methods of Section 3 and fixing $g(u, c)=-u c^{2}$, we may transform the generalised model to obtain the following travelling wave equations

$$
\begin{aligned}
\frac{1}{a}\left(2 U C^{2} \chi(C)-a^{2}\right) \frac{d U}{d z} & =f(U, C)-\frac{U^{3} C_{2}}{a^{2}} \frac{d}{d C}\left(C^{2} \chi(C)\right), \\
\frac{d C}{d z} & =\frac{U C^{2}}{a} .
\end{aligned}
$$

We note that this system possesses a singular barrier $\mathrm{S}$ (when the left hand side of (4.1) is zero) and a $U$ nullcline $\mathrm{N}$ (when the right hand side of (4.1) is zero).

Applying the Rankine-Hugoniot conditions to the conservation form of our model, it is possible to show further that this model admits blunt form travelling wave solutions that satisfy the Lax entropy condition and for which $U$ jumps directly to zero from the jump curve $J$ where

$$
J: U=\frac{a^{2}}{C^{2} \chi(C)} .
$$

For comparison with our earlier work, and in order to illustrate the effect of a nonlinear haptotaxis coefficient on our generalised model, we now fix

$$
f(U, C)=U(1-U) \quad \text { and } \quad \chi(C)=\frac{C^{4}}{\left(1+C^{5}\right)^{2}} .
$$

In Figures 13 and 14 we plot the curves $\mathrm{S}, \mathrm{N}$ and $\mathrm{J}$ for two values of the wavespeed $a$. In both cases there is one hole in the wall $H$ in the positive quadrant of the $(U, C)$ phase plane at which $\mathrm{S}$ intersects $\mathrm{N}$.

As with the models already described in this paper, there is a smooth phase plane connection from the malignant steady state $(U, C)=(1,0)$ to the hole in the wall $H$. This trajectory then crosses the singular barrier into the region where $d U / d z<0$ and $d C / d z>0$. The remaining question is what 


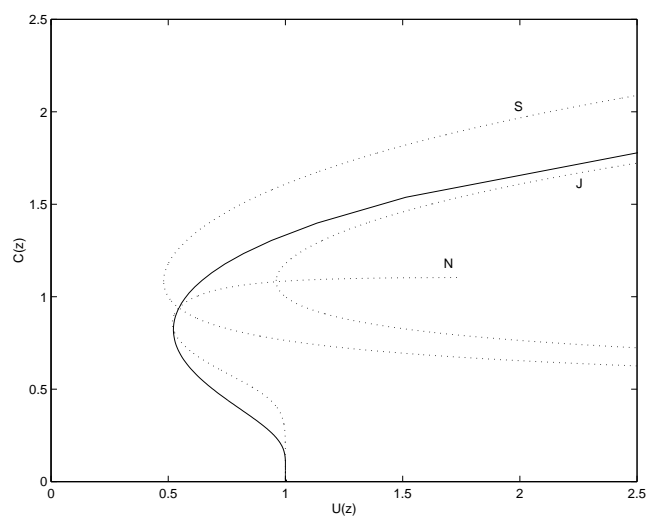

FIG. 14. A plot of the phase plane behaviour for (4.1)-(4.2) with $\chi(C)$ defined by (4.4) and $a=0.5$. The curves $\mathrm{S}$ and $\mathrm{N}$ intersect once $H$, with $\mathrm{S}$ lying between the malignant steady state $(U, C)=(1,0)$ and the curve $\mathrm{J}$. The plot shows a trajectory leaving $(U, C)=(1,0)$, and crossing the singular barrier at $\mathrm{H}$. Unlike Figure 13 (when $a=0.365$ ) this trajectory does not cross $\mathrm{J}$ and, therefore, no travelling wave solutions with a blunt $U$ wave-front exist for this value of $a$.

happens to this trajectory once it has crossed S. In particular, does it cross the curve $\mathrm{J}$ and then complete a blunt interface connection to a healthy steady state $(U, C)=(0, \hat{c})$ ?

The modified haptotactic function causes the gradient $d C / d U$ of the curve $\mathrm{N}$ to become positive on this side of the singular barrier. Therefore for each of the values of $a$ the trajectory (which initially has $d C / d U<0$ ) soon crosses $\mathrm{N}$ for a second time, after it has passed through $H$. The gradient $d U / d z$ is positive thereafter leading to non-monotonic $U(z)$ profiles. For small values of $a$ the trajectory then crosses $\mathrm{J}$ twice, meaning that there are two values of $\hat{c}$ for which blunt profile solutions exist with this value of $a$. There is a critical value of $a$ for which the trajectory intersects $\mathrm{J}$ tangentially and for all values of $a$ greater than this critical value there is no intersection between the trajectory and J. This leads again to a biphasic relationship between $a$ and $\hat{c}$.

The results from numerical solutions to the PDEs using the Lax Friedrichs scheme are consistent with this phase plane behaviour. Initial data where $u_{0}(x)$ has semi-compact support evolves to travelling wave solutions with blunt $u(x, t)$ interfaces at the front of the wave. Figure 15 shows how the wavespeed of such solutions behaves in a biphasic manner as $\hat{c}$ is varied. In Figure 16 we plot the profiles of a typical numerical solution of (4.1)-(4.2). The blunt front and non-monotonicity of the $U$ profile can be seen. Anderson et al. (2000) obtained similar numerical profiles from their model of malignant invasion which combined haptotaxis and random motion. However, the higher order of their equations prevented them from deriving analytical insight into the origins of the non-monotonic profile.

Our brief investigation of the modified model illustrates that the existence of multiple holes in the wall is not necessary for obtaining a biphasic relationship between the wave speed $a$ and the farfield ECM concentration $\hat{c}$. Future work will involve establishing how the number of blunt form travelling wave solutions that such models admit depends on the number of (physically relevant) holes in the wall and the structure of the jump curve J.

There are naturally many ways in which our simple model of malignant invasion could be extended. For example, it could be combined with the models developed by Gatenby \& Gawlinski (1996) to investigate the additional effect that local $\mathrm{pH}$ levels have on the invasion process. Alternatively, it could be combined with the models developed by Chen et al. (2001) and Roose et al. (2003) to study the manner in which the mechanical properties of the healthy tissue influence tumour invasion. Equally, 


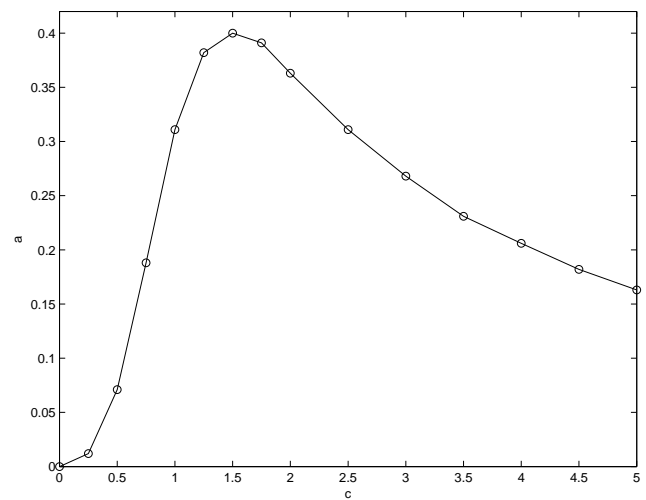

FIG. 15. A plot of the wavespeed, $a$, of travelling wave solutions to the PDE model evolving from semi-compact initial data, against the far-field concentration of connective tissue $\hat{c}$, for $\chi(c)$ defined in (4.4). There is a biphasic relationship between $a$ and $\hat{c}$.

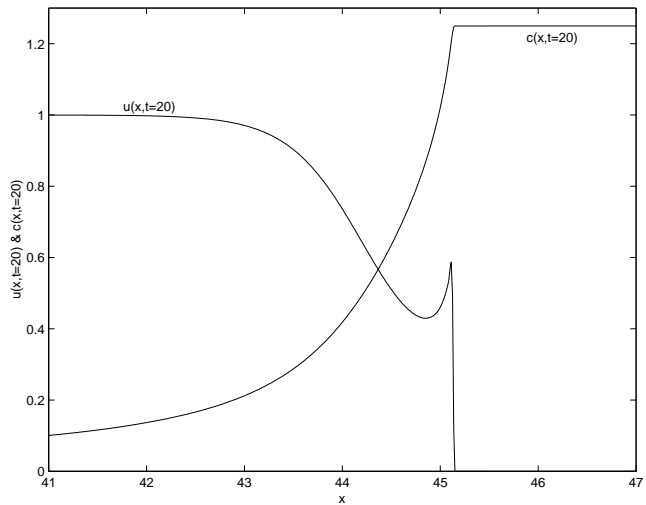

FIG. 16. Typical travelling wave profiles of tumour cells $u(x, t=20)$ and connective tissue $c(x, t=20)$ obtained via the numerical solution of the PDEs with haptotactic coefficient (4.4). This solution, with the blunt wave-front for $u(x, t=20)$, evolves from semi-compact initial conditions (3.21)-(3.22). Observe the non-monotonicity of the $u$ profile. For this solution $\hat{c}=1.25$ and $\chi(c)$ is defined in (4.4)

by incorporating some of the detailed biochemistry described in the introduction and by focussing on specific cancers, it should be possible to extend and specialise the model so that it generates better testable predictions. For example, we might focus on the role of the metalloprotease MMP-2 and its inhibitor TIMP-2 in the invasion and progression of breast carcinoma (Shiomi \& Okada (2003)).

In spite of its simplicity, our mathematical model is able to reproduce several key features of malignant invasion studied in vitro. We now explain how our model may have wider practical implications. First, our results regarding the biphasic dependence of tumour invasion on the concentration of the host tissue may explain why certain tumours metastasize preferentially to specific organs: secondary tumours are likely to develop in distant organs if the concentration of the tissue matrix there is such that the invasion speed of any tumour fragments that colonise the tissue is the same as (or greater than) that at the site of the primary tumour. Thus by performing in vitro experiments to construct the biphasic curve showing how invasion wavespeed varies with ECM density or composition and combining these results 
with measurements of the ECM composition of other organs it should be possible to predict those tissues through which the tumour cells will spread most readily: these predictions could then be tested by monitoring the sites at which metastases appear.

Our results may also provide insight into the limited success with which protease inhibitors have been able to arrest tumour invasion. The principle underlying this treatment is that the inhibitors will neutralise proteases produced by the tumour cells and leave the surrounding healthy tissue intact (StetlerStevenson et al. (1993)). For example, in one family of Apc ${ }^{\min }$ mice overexpression of TIMP1 had no effect on tumour development although it enhanced intestinal adenoma formation in another family, the latter family experiencing slower growth when treated with a different metalloprotease inhibitor (Heppner-Goss et al. (1998)). From Figure 10 it is clear that if, in the absence of treatment, the underlying tissue concentration is such that the speed of invasion increases as the tissue concentration increases, then a treatment which increases the concentration of the tissue may actually increase the speed with which the tumour invades. More constructively, given estimates of model parameters such as the concentration of the healthy tissue into which a tumour is invading and its doubling rate, our model could be used to identify those patients who are most likely to benefit from treatment with protease inhibitors. In particular, we predict that patients whose tumours are such that the models parameters reside on the increasing branch of a biphasic curve would benefit from treatment with tissue-degrading proteases whereas those on the decreasing branch would benefit from treatment with protease inhibitors - in both cases we would expect a reduction in the speed of tumour invasion.

\section{Acknowledgements}

Figures 1 and 2 were reprinted from the European Journal of Cancer, Volume 35, Perumpanani AJ and Byrne HM, Extracellular matrix concentration exerts selection pressure on invasive cells, Pages 12741280, Copyright(1999) with permission from Elsevier. The authors gratefully acknowledge the support of the EPSRC through a studentship award (BPM) and an Advanced Research Fellowship (HMB). BPM was a member of The Centre for Mathematical Biology, Mathematical Institute, Oxford when much of this work was carried out.

\section{REFERENCES}

Abramowitz, M. \& Stegun, I.A. (EDITORS) (1965) Handbook of Mathematical Functions. New York: Dover Publications Inc.

Anderson, A.R.A., Chaplain, M.A.J., Newman E.L., Steele R.J.C. \& Thompson A.M. (2000) Mathematical modelling of tumour invasion and metastasis, Journal of Theoretical Medicine, 2 (2), 129-154.

ANDERSON, A.R.A. (2005) A hybrid mathematical model of solid tumour invasion: the importance of cell adhesion, Math. Med. Biol., 22 (2), 163-186.

Aznavoorian, S., Stracke, M.L., Krutzsch, H., Schiffman, E. \& Liotta, L.A. (1990) Signal transduction for chemotaxis and haptotaxis by matrix molecules in tumour cells, J. of Cell Biology, 10 (4), 1427-1438.

BAiley, H. \& Love, R.J. (1995) Bailey and Loves Short Practice of Surgery, 23rd edition. London: Arnold.

CAirns, R.A., KHOKHA, R. \& Hill, R.P. (2003) Molecular mechanisms of tumour invasion and metastasis: an integrated view, Curr. Mol. Med., 3 (7), 659-691.

CHANG, C. \& WeRB, Z. (2001) The many faces of metalloproteases: cell growth, invasion, angiogenesis and Metastasis, Trends Cell Biol., 11, S37-S43.

Chen, C.Y., Byrne, H.M. \& King, J.R. (2001) The influence of growth-induced stress from the surrounding medium on the development of multicell spheroids. J. Math. Biol., 43 (3), 191-200.

Courant, R. \& Hilbert, D. (1962) Methods of Mathematical Physics II. New York: Interscience.

GATENBy, R.A. \& GAWLINSKi, E.T. (1996) A reaction-diffusion model of cancer invasion. Cancer Res., 56 (24), 
$5745-5753$

HePPNER-Goss, K.J., BRown, P.D. \& MATRISiAn, L.M. (1998) Differing effects of endogenous and synthetic inhibitors of metalloproteainases on intestinal tumorigenesis, Int. J. Cancer, 78 (5), 629-635.

HUNDSDORFER, W. \& VERWER, J.G. (2003) Numerical solution of time-dependant advection-diffusion reaction equations. Springer Series in Computational Mathematics, 33. New York: Springer Verlag.

LACEY, A.A., OCKENDON, J.R. \& TAYLOR, A.B. (1982) Waiting time solutions of a nonlinear diffusion equation, SIAM J. Appl. Math., 42 (6), 1252-1264.

Landman, K.A., Pettet, G.J. \& Newgreen, D.F. (2003) Chemotactic cellular migration: smooth and discontinuous travelling wave solutions, SIAM J. Appl. Math., 63 (4), 1666-1681.

LEVeque, R.J. (1992) Numerical Methods for Conservation Laws, 2nd edition. Basel: Birkhauser Verlag.

LiotTA, L.A. (1986) Tumour invasion and metastasis: role of the extracellular matrix, Cancer Research., 46 (1), $1-7$.

MARChANT, B.P. \& NORBURY, J. (2002) Discontinuous travelling wave solutions for certain hyperbolic systems, IMA J. Appl. Math., 67 (2), 201-224.

Marchant, B.P., Norbury, J. \& Perumpanani, A.J. (2000) Travelling shock waves arising in a model of malignant invasion, SIAM J. Appl. Math., 60 (2), 463-476.

MARChANT, B.P., NORBURY, J. AND ShERRATT, J.A. (2001) Travelling wave solutions to a haptotaxis-dominated model of malignant invasion, Nonlinearity, 14 (6), 1653-1671.

Orme, M.E. \& Chaplain, M.A.J. (1996) A mathematical model of vascular tumour growth and invasion, Math. Comp. Modelling, 23 (10), 43-60.

Perumpanani, A.J. \& B yrne, H.M. (1999) Extracellular matrix concentration exerts selection pressure on invasive cells, European Journal of Cancer, 35 (8), 1274-1280.

Perumpanani, A.J., Norbury, J., Sherratt, J.A. \& Byrne, H.M. (1996) Biological inferences from a mathematical model for malignant invasion, Invasion and Metastasis, 16 (4-5), 209-221.

Perumpanani, A.J., Norbury, J., Sherratt, J.A. \& B yrne, H.M. (1999) A two parameter family of travelling waves with a singular barrier arising from the modelling of matrix mediated malignant invasion, Physica $D, 126$ (3-4), 145-159.

Pettet, G.J., McElwain, D.L.S. \& Norbury, J. (2000) Lotka-Volterra Equations with Chemotaxis: walls, barriers and travelling waves, IMA J. Math. Appl. Med. Biol., 17 (4), 395-413.

Roose, T., Netti, P.A., Munn, L.L., Boucher, Y. \& Jain, R.K. (2003) Solid stress generated by spheroid growth estimated using a linear poroelasticity model, Microvasc. Res., 66 (3), 204-212.

SherRATT, J.A. \& NowAK, M.A. (1992) Oncogenes, anti-oncogenes and the immune response to cancer: a mathematical model, Proc. Roy. Soc. Lond. B, 248 (1323), 261-271.

Shiomo, T. \& OKADA, Y. (2003) MT1-MMP and MMP-7 in invasion and metastasis of human cancers, Cancer Met. Rev., 22 (4), 145-152.

Sidenius, N. \& Blasi, F. (2003) The urokinase plasminogen activator system in cancer: recent advances and implication for prognosis and therapy, Cancer Met. Rev., 22 (2-3), 205-222.

Stetler-Stevenson, W.G., Aznavoorian, S. \& Liotta, L.A. (1993) Tumour cell interactions with the extracellular matrix during invasion and metastasis, Ann. Rev. Cell Biol., 9, 541-573.

Tsuboi, R. \& RifKIN, D.J. (1990) Bimodal relationship between invasion of the amniotic membrane and plasminogen activator activity, Int. J. Cancer, 46 (1), 56-60. 


\section{Figure Captions}

1. Experimental results reproduced from Perumpanani \& Byrne (1999) illustrating the biphasic relationship between tumour invasiveness and the concentration of collagen. Dose response curves of HT1080 invasion distance are shown for various concentrations of Type I collagen.

2. Experimental results reproduced from Perumpanani \& Byrne (1999) illustrating the biphasic relationship between tumour proliferation and the concentration of collagen. Dose response curves for HT1080 proliferation are shown for various concentrations of Type I collagen. Proliferation is measured in Reference Fluorescence Units (RFUs).

3. A phase plane connection and a solution profile for the blunt interface travelling wave solution of (2.7)-(2.8) for $a=0.5$ and $k=0$. In the solution profiles, the blunt interface in the $U$ profile can be clearly seen as can the corresponding corner in the $C$ profile.

4. The $U$ and $C$ nullclines and the jump curve, denoted $\mathrm{N}, \mathrm{S}$ and $\mathrm{J}$ respectively, of the regularised system when $k=0.3<k^{*}$ and $a=0.9$. The $C$ nullcline coincides with the singular barrier $\mathrm{S}$ of the original, singular system. The arrows indicate the vector field directions across the nullclines. Note the effect of $k$ in the top left hand corner of the diagram. 
5. A sketch showing how the positive solutions $C_{H}$ of (3.9) depend upon $k$ for a fixed value of $a$. Parameter values: $a=0.9, k^{*}=0.37$.

6. A plot of a trajectory (the solid line) leaving the steady state $\mathrm{H}_{1}$ of the regularised system when $k=0.3$ and $a=0.7$. The trajectory intersects the $\mathrm{J}$ curve twice; first when $C=1.415$ and second when $C=7.290$.

7. A plot of a trajectory (the solid line) leaving the steady state $\mathrm{H}_{1}$ of the regularised system when $k=0.3$ and $a=0.9$. The curves $\mathrm{J}$ and $\mathrm{S}$ are unchanged, whereas $\mathrm{N}$ is changed, especially near the $C$ axis.

8. An illustration of the continuity argument which demonstrates that $\hat{c}_{\text {max }}$ increases with $k$. The singular barrier/ $C$ nullcline and $\mathrm{J}$ curve for $a=0.9$ are shown along with the corresponding $U$ nullclines for $k=0.1,0.2$. The hole in the wall $\mathrm{H}_{1}$ moves from right to left as $k$ is increased. The trajectories leaving $\mathrm{H}_{1}$ are also marked. $d C / d U$ becomes less negative as $k$ is increased and thus the $C$ value where the trajectories cross $\mathrm{J}$ increases.

9. A plot showing the evolution of the $u(x, t)$ profile of a Lax Friedrichs solution to (2.7)-(2.8) from smooth semi-compact initial data of the form (3.21)-(3.22) for $k=0$ and $\hat{c}=1.0$. 
10. A plot of the wavespeed $a$ of travelling wave solutions to (2.7)-(2.8) (evolving from semi-compact initial conditions) against the far-field concentration $\hat{c}$ of connective tissue when $k=0.3$. These wavespeeds were approximated from Lax Friedrichs numerical solutions to (2.7)-(2.8).

11. Travelling wave profiles of $u(x, t)$ and $c(x, t)$ resulting from numerical solution of (2.7)-(2.8) with compact initial data (3.21)-(3.22) for $\mathrm{k}=0.3$ and $\hat{c}=1.415$. The measured wavespeed of the solution profiles was $a=0.695$.

12. Travelling wave profiles of $u(x, t)$ and $c(x, t)$ resulting from numerical solution of (2.7)-(2.8) with compact initial data (3.21)-(3.22) for $\mathrm{k}=0.3$ and $\hat{c}=7.290$. The measured wavespeed of the solution profiles was $a=0.702$.

13. A plot of the phase plane behaviour for (4.1)-(4.2) with $\chi(C)$ defined by (4.4) and $a=0.365$. The singular barrier $\mathrm{S}$ and the $U$ nullcline $\mathrm{N}$ intersect once at the hole in the wall $H$. The singular barrier lies between the malignant steady state $(U, C)=(1,0)$ and the curve $\mathrm{J}$. The plot shows a trajectory leaving $(U, C)=(1,0)$, crossing the singular barrier at $\mathrm{H}$ and intersecting the curve $\mathrm{J}$ twice. From each intersections with $\mathrm{J}$ the trajectory may complete the travelling wave solution by jumping down to $U=0$, with a blunt wave-front. 
14. A plot of the phase plane behaviour for (4.1)-(4.2) with $\chi(C)$ defined by (4.4) and $a=0.5$. The curves $\mathrm{S}$ and $\mathrm{N}$ intersect once $H$, with $\mathrm{S}$ lying between the malignant steady state $(U, C)=(1,0)$ and the curve $\mathrm{J}$. The plot shows a trajectory leaving $(U, C)=(1,0)$, and crossing the singular barrier at H. Unlike Figure 13 (when $a=0.365$ ) this trajectory does not cross $\mathrm{J}$ and, therefore, no travelling wave solutions with a blunt $U$ wave-front exist for this value of $a$.

15. A plot of the wavespeed, $a$, of travelling wave solutions to the PDE model evolving from semicompact initial data, against the far-field concentration of connective tissue $\hat{c}$, for $\chi(c)$ defined in (4.4). There is a biphasic relationship between $a$ and $\hat{c}$.

16. Typical travelling wave profiles of tumour cells $u(x, t=20)$ and connective tissue $c(x, t=20)$ obtained via the numerical solution of the PDEs with haptotactic coefficient (4.4). This solution, with the blunt wave-front for $u(x, t=20)$, evolves from semi-compact initial conditions (3.21)(3.22). Observe the non-monotonicity of the $u$ profile. For this solution $\hat{c}=1.25$ and $\chi(c)$ is defined in (4.4). 